\title{
MANIPULATION OF WHOLE BLOOD USING TRAVELING WAVE DIELECTROPHORESIS
}

\author{
Y.J. Lo, A.M. Wo, and U. Lei \\ Institute of Applied Mechanics, National Taiwan University, Taipei, Taiwan
}

\begin{abstract}
This paper presents new results on manipulation of undiluted whole blood using traveling wave dielectrophoresis (twDEP). Although twDEP is not new, data on whole blood manipulation is rare. The twDEP in this wotk consists of four $2 \mathrm{D}$ electrodes of $20 \mu \mathrm{m}$ width operating at $10 \mathrm{MHz}$ sinusoidal wave with 90 degree phase apart. Results show that whole blood bulk flow can be manipulated at-will in either direction. Moreover, simultaneous bi-direction motion is also observed at different height of the $25 \mu \mathrm{m}$ flow channel. This flow feature might be utilized for separation purpose.
\end{abstract}

\section{INTRODUCTION}

As is well known, a dielectrophoretic (DEP) force is induced in a non-uniform electric field to achieve manipulation of particles within a medium, accounting for dielectric and conductive properties of both particles and the medium. Many researchers have explored DEP and, its counterpart, twDEP for manipulation and separation of biological particles, cells, protein, virus, and even DNA [1-6]. Although twDEP is not new, data on whole blood manipulation is rare. This paper presents manipulation of whole blood using twDEP as shown in Fig. 1.

Figure 2 sketches the micro-channel with inter-digitated, parallel electrode at electro-potential of 90 degrees apart - either $0,90,180$, and 270 degrees or $270,180,90$, and 0 degrecs. Three electrode configurations were tested: (1) $20 \mu \mathrm{m}$ width and $20 \mu \mathrm{m}$ gap, (2) $20 \mu \mathrm{m}$ width and $30 \mu \mathrm{m}$ gap, and (3) $20 \mu \mathrm{m}$ width and $50 \mu \mathrm{m}$ gap. The channel, with height of $25 \mu \mathrm{m}$, was fabricated using SU-8 as photoresist and soft lithographic technique. The electrodes were e-beam deposited using chromium. A fixed frequency of $10 \mathrm{MHz}$ is applied to all electrodes, which corresponds to non-negligible contributions from both conventional DEP and twDEP on the Clausius Mossotti diagram.

\section{THEORY}

\section{Physical Model}

When a dielectric particle is immersed in a dielectric medium under the influence of a non-uniform electric field, a particle and the medium are polarized to a different degree. Therefore, a force exerting on the particle is induced as a result of the phenomenon of the induced dipole interacting with electric field. The time-mean force [1] is described as

$\left.\vec{F}_{\mathrm{dep}}=2 \pi \varepsilon_{0} \varepsilon_{m} r^{3}\left(\begin{array}{l}\operatorname{Re}[K(\omega)] \nabla E_{r s}^{2}+ \\ \operatorname{Im}[K(\omega)]\left(E_{x 0}^{2} \nabla \phi_{x}+E_{y 0}^{2} \nabla \phi_{y}+E_{20}^{2} \nabla \phi_{z}\right)\end{array}\right)\right)$

where $\varepsilon_{0}$ is the permittivity of vacuum, $\varepsilon_{m}$ the relative permittivity of medium, $E_{r m s}$ the root-mean-square of the electric field, $r$ the radius of cells, and $K(\omega)$ the so-called Clausius-Mossotti factor (C.M. factor).

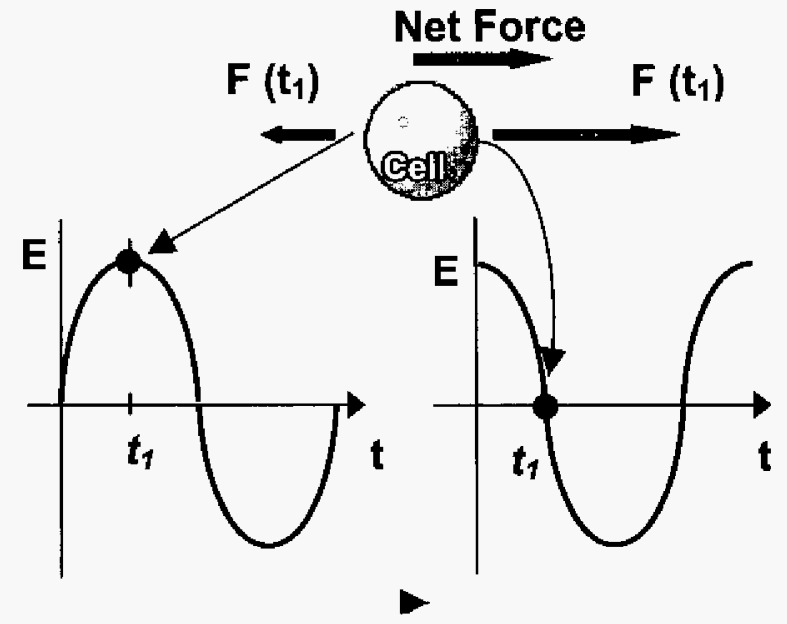

Fig. 1. Sketch of twDEP force on a cell. The twDEP force is due to spatial variation of the phase of electro-potential acting on the cell.

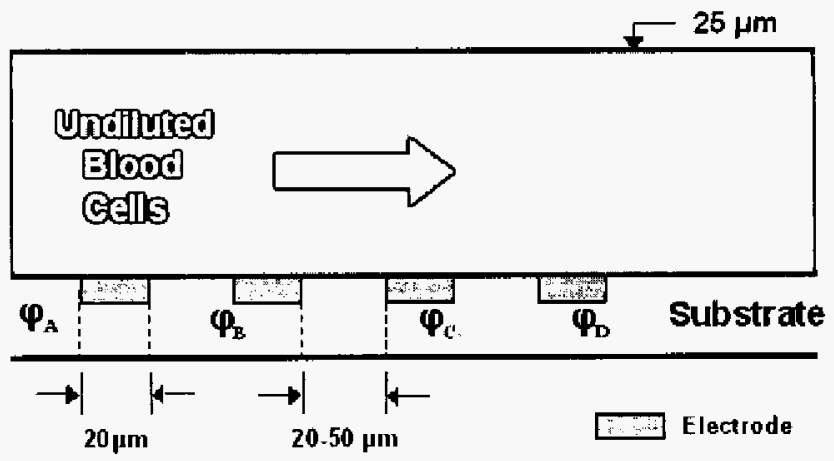

Fig. 2. Sketch of the present device. Traveling wave DEP pumps undiluted whole blood through the micro-channel with 90 degrees phase shift between adiacent electrodes. 
This factor related with cell and medium electric properties as follows,

$K(\omega)=\left(\frac{\varepsilon_{p}^{*}-\varepsilon_{m}^{*}}{\varepsilon_{p}^{*}+2 \varepsilon_{m}^{*}}\right)$,

where the superscript ${ }^{*}$ is the complex permittivity ( $\varepsilon^{*} \equiv \varepsilon-j \sigma / \omega, \sigma$ is the conductivity of material, and $\omega$ the angular velocity), and subscript $p$ and $m$ denote particle and medium respectively. Through a double layer method, the electric field acting on a RBC can be acquired. Thus, the Clausius-Mossotti factor also can be calculated and is shown in Fig. 3. The equation has two parts: the first term resulted called conventional dielectrophoresis (CDEP) is associated with real part of Clausius-Mossotti factor and non-uniformly spatial electric field intensity. The second term attributed to imaginary part of Clausius-Mossotti factor and non-uniform phase distribution is named after traveling wave dielectrophoresis (twDEP). The Clausius-Mossotti diagran suggests that cDEP effect exists at all frequencies but not twDEP. The twDEP contributes merely at frequency ranging from $1 \mathrm{Mhz}$ to $1 \mathrm{Ghz}$.

\section{System Interpretation}

The relationship between the applied electric field and the induced dipole moment $k(\omega)$ is presented in Fig. 4a for the amplitude and Fig. $4 \mathrm{~b}$ for the phase. Its physical for frequency meaning can be understood as follows. From the figure $4 \mathrm{~b}$, it can be seen that the dipole moment changes sign. from $1 \mathrm{MHz}$ to $1 \mathrm{GHz}$, In the other words, the cDEP force changes direction; the system possess a 180 degree phase lag. In the same frequency range, twDEP works due to the non-uniformly spatial phase distribution with the 180 degree transition of dipole moment. Thus, these two phenomena can be exploited for cell manipulation.

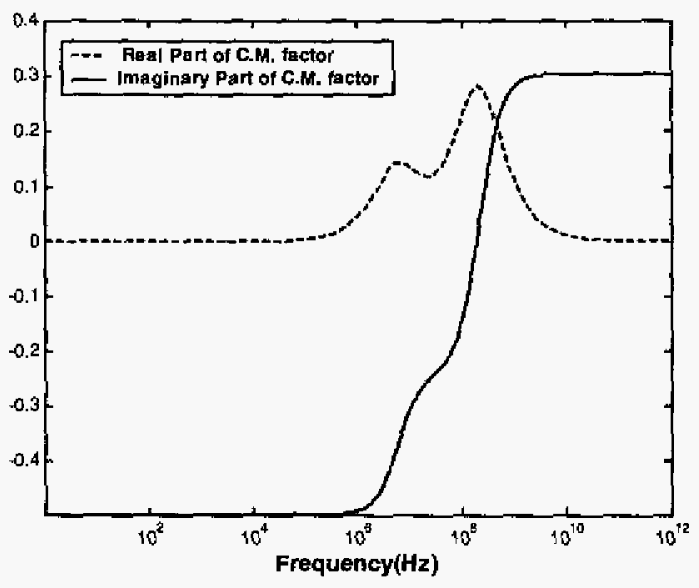

Fig. 3. Calculated result of Clausius-Mossotti factor obtained from considering of the electric double layer of a cell. a)

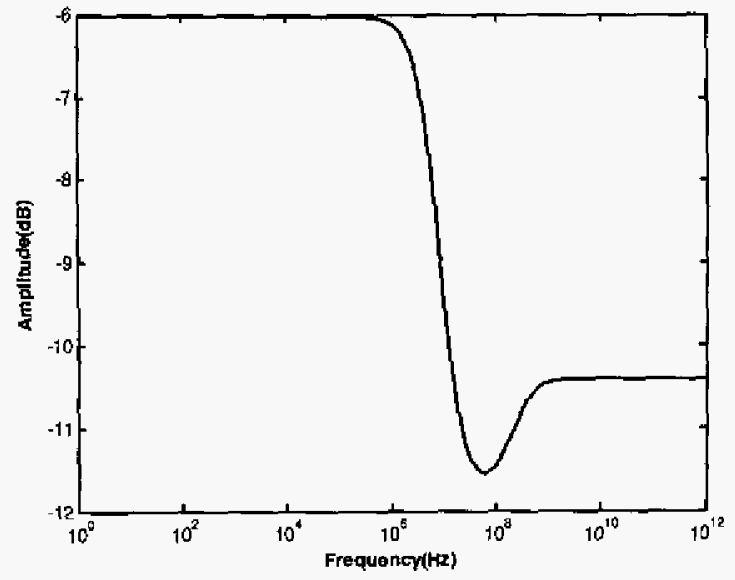

b

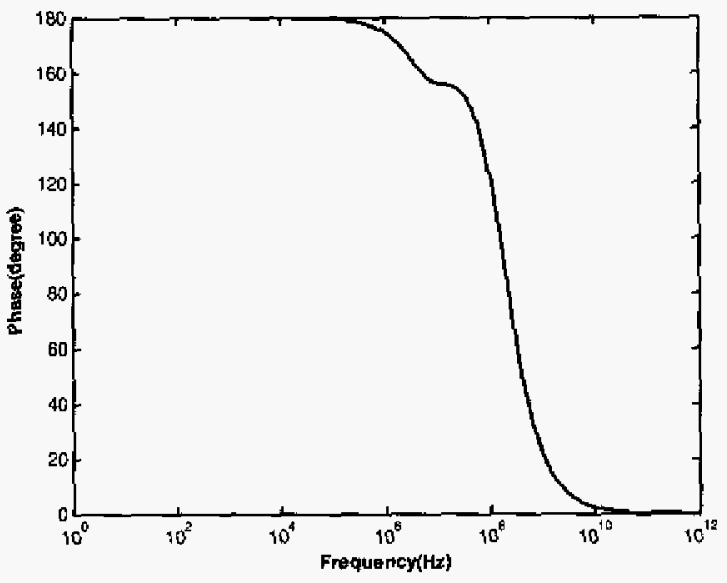

Fig. 4. The (a) amplitude and (b) phase of the Clausius-Mossotti factor, as shown in Fig. 3.

\section{Fabrication}

\section{Electrode}

Fabrication of the electrodes is as follow. The first step is to clean the glass (15mm length and $52 \mathrm{~mm}$ width) and substrate surface with a mixture of sulfuric acid and hydrogen peroxide with the proportion of $1: 3$. Well-preparation substrates are loaded into the e-beam evaporator for a $2000 \AA$ chromium or gold deposition. A two-step deposition program is used to ensure good conductive and adhesion property of film. Development transforms the latent image formed during exposure into a relief image, and thus, it dominates the photolithography process. Then, immerse the exposed elements in MF-319 for 1 minute. Afterwards, it is placed into a furnace $120 \mathrm{C}$ for 5 minutes. After cooling, the substrate is immersed in the $\mathrm{Cr}$ etchant for 5 to 6 minutes depending, on the uniformity of deposited $\mathrm{Cr}$. Finally, the protective PR layer is removed using acetone.

\section{Micro-channel}

The micro-channel is fabricated with SU8-50 and replica molded with PDMS. The fabrication of SU8 mold is similar to that of electrode. In order to coerce all the cells with sufficient electric field strength, we design a $25 \mu \mathrm{m}$ 
height channel which is larger than the cell size ranging from 5 to $15 \mu \mathrm{m}$. In this requirement, SU8 layer is achieved by spinning 4000 RPM for 30 seconds, which achieves a $25 \mu \mathrm{m}$ thickness.

\section{Device bonding}

After the fabrication of micro-channel and electrodes, oxygen plasma bonding is used to integrate the two independent sub-devices. First, the surfaces are cleaned for bond, and DI water and acetone are used to wash the surfaces and dry via compressed air. Then, the cleaned surfaces are placed horizontally inside the plasma cleaner, outer door closed, and the suction pump is activated with the plasma cleaner off. After about 2 minutes, the pressure within the plasma cleaner reaches about 300 milli-torr. Then we turn the plasma cleaner (Harrick Scientific) on high power, and a white-colored glow will appear. Air is allowed to flow into the plasma chamber through a fine valve connect to the lid until the pressure rises to 1 to 2 torr, then the valve is closed. A bright reddish glow will result. After about 30 seconds, the plasma cleaner is turned off and air is allowed to flow into the chamber until atmospheric pressure is reached. Open plasma chamber and pick up the two sub-devices. Bond them together in a microscope in a minute and the integration is completed.

\section{Results and Discussion}

\section{Cell velocity}

Figure 3 presents micrographs of steady-state motion of the whole blood cells under two sets of spatial phase variation. Figures $5 \mathrm{a}$, with electro-potential phase at 270 , 180,90 , and 0 degrees, shows the cells are moving, as a bulk, towards the direction of increasing. Figure $5 \mathrm{~b}$ also confirms this effect by reversing the phase $-0,90,180$, and 270 degrees - and the motion of the cells reverse. Figure 4 presents the averaged cell velocity, obtained by tracking 15 cells over an uniformly spaced domain, with variation in electrode gap at different applied voltages. Results confirm that cell velocity increases with stronger electro-potential gradient (smaller gap). In a low Reynolds number, drag varies linearly with cell velocity, and twDEP force is related to square of applied voltage. The curve of average cell velocity to applied voltage is parabolic correspondent to the theoretic analysis.

\section{Bi-directional manipulation}

Traveling wave DEP can give rise to simultaneous bi-direction motion on cells, as shown in Fig. 7 . The cells exhibit a motion where they move in opposite direction depending on the height normal to the electrodes. This can be understood from the spatial force distribution, due to twDEP alone, computed by solving the time averaged force equation. Figure 8 shows the time-averaged twDEP force vectors along ( $x$-dir.) and normal to the electrodes (z-dir), with expanded view shown (blue vectors). Results show at height less than approximately $5 \mu \mathrm{m}$ from the plane of the electrodes, the net force is in the opposite direction from that at further distance away. However, in our experiment, after some time (steady state), conventional DEP (negative) force causes the cells to be sufficiently repelled from the electrodes so that they exhibits behavior as shown in Fig. 3. Nevertheless, at higher frequency, twDEP dominates over conventional $D E P$ and would resuit in steady-state bi-directional motion, which might be harvested as a new method of sorting particles/cells.

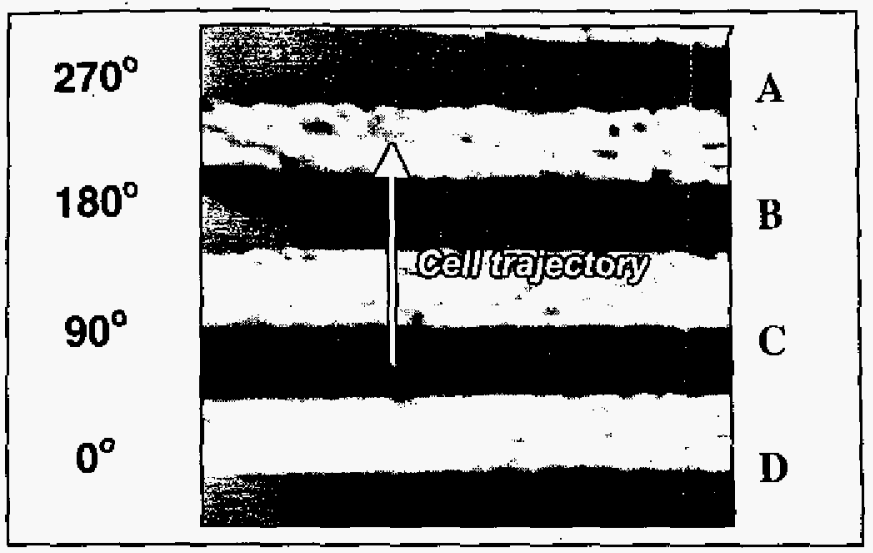

Fig. 5a. Micrograph of steady-state motion of whole blood cells being pumped by twDEP with phasing between electrodes as shown on right.

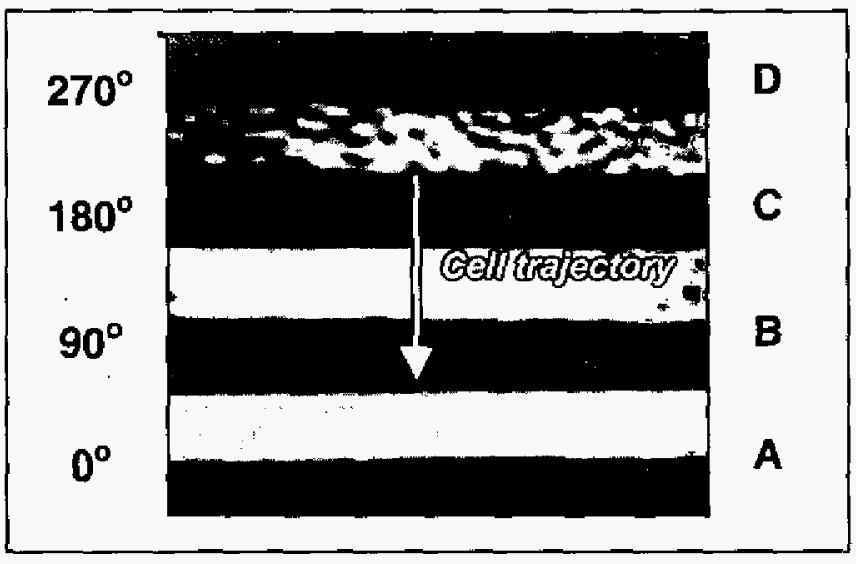

Fig. 5b. Micrograph of steady-state motion of whole blood cells being pumped by twDEP with phasing between electrodes as shown on right.

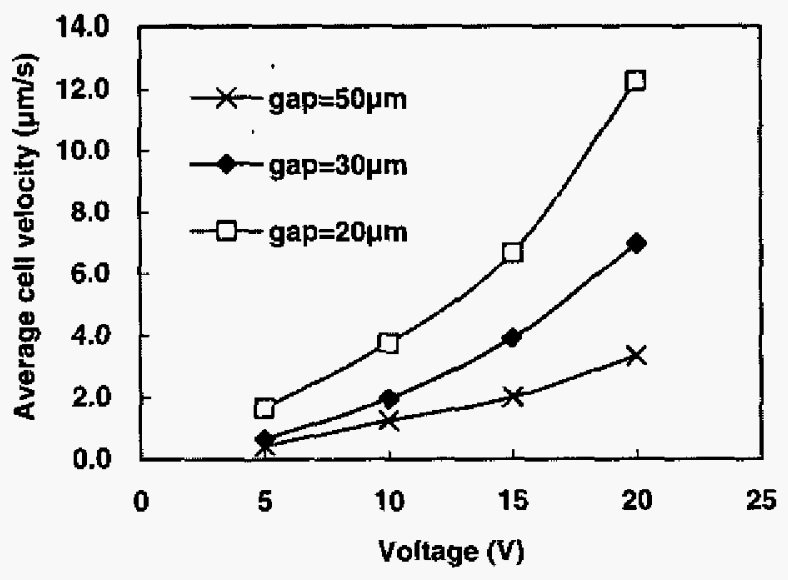

Fig. 6. Effect of electrode gap and voltage on average cell velocity. The width of each electrode is $20 \mu \mathrm{m}$. 


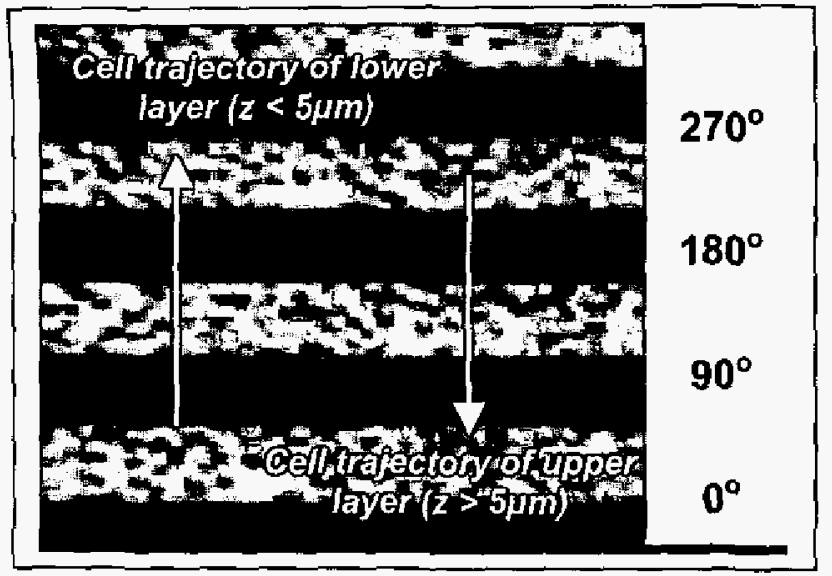

Fig. 7. Bi-directional cell motion due to spatial variation of twDEP force normal to the plane of electrode (see Fig. 8).

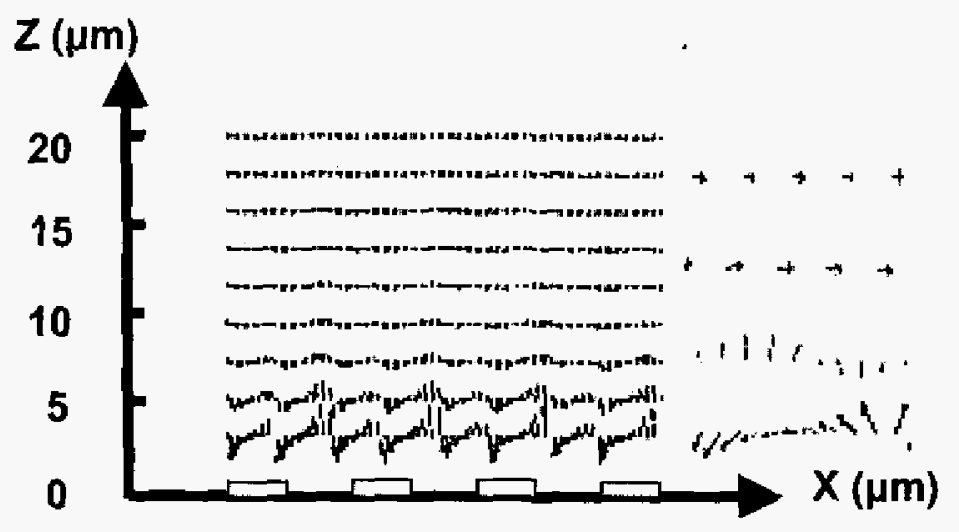

Fig. 8. Computational result of twDEP force vectors ( $x$ : along the electrodes parallels to cell motion; z: normal to the plane of electrode). Blues arrows on the right show enlarged view. Below $Z<5 \mu m$ (approx.), force vectors (blue arrows) are to the left (except for single vector on the far right due to edge effect). Above $Z>5 \mu \mathrm{m}$ (approx.), force vectors (blue arrows) are to the right. This directional variation of twDEP force causes bi-directional cell motion.

\section{Conclusion}

This work studied manipulation of undiluted whole blood using traveling wave dielectrophoresis (twDEP). The twDEP in this work consists of four 2D electrodes of $20 \mu \mathrm{m}$ width operating at $10 \mathrm{MHz}$ sinusoidal wave with 90 degree phase apart. The effect of different gap distance between electrodes and variation in electrode voltages are also studied.

Results show that whole blood bulk flow can be manipulated at-will in either direction. The average cell velocity depends on the electrode gap distance and voltage - the smaller the gap and the larger the voltage give rise to greater cell velocity. Moreover, simultaneous bi-direction motion is also observed at different height of the $25 \mu \mathrm{m}$ flow channel. This flow feature might be utilized for separation purpose.

\section{Reference:}

[1] T,B. Jones, Electromechanics of Particles, Cambridge University Press, 1995.

[2] Michael Pycraft Hughes, "Micro- and Nanoelectrokinetics in Medicine," IEEE Engineering in Medicine and Biology Magazine, Nov.-Dec. 2003, p. 3.

[3] Muller, T, "Potential of Dielectrophoresis for Single Cell Experiment," IEEE Engineering in Medicine and Biology Magazine, Nov.-Dec. 2003, pp. 51-61.

[4] N. G . Green, and H. Morgan, "Microdevices for Dielectrophoretic Flow- through Cell Separation," IEEE Engineering in Medicine and Biology Magazine, Nov.-Dec. 2003, pp. 85-90.

[5] K. Pant, J. Feng, G. Wang, S. Krishnamoorthy, and S. Sundaram, "Separation of Bioparticulate Matter Using Traveling Wave Dielectrophoresis," Proceedings of Micro TAS 2003, pp. 1207-1210.

[6] M.P. Hughes, "Dielectrophoresis in Laboratories-on-a-Chip," (invited review) Electrophoresis, Vol. 23, pp. 2569-2582, 2002. 